\title{
Test-Retest Reliability and Validity of a Child and Parental Questionnaire on Specific Determinants of Cycling to School
}

\author{
Fabian Ducheyne, Ilse De Bourdeaudhuij, \\ Matthieu Lenoir, and Greet Cardon \\ Ghent University
}

\begin{abstract}
This study examined the reliability of a newly developed child and parental questionnaire on specific determinants of cycling to school among 10-12 year olds. Validity of child reported distance, bicycle equipment and basic bicycle skills was also investigated. In total 211 children and 33 parents participated in this study. The reliability of the questionnaires was acceptable with results indicating reliability ranging from fair to perfect agreement. Therefore, the questionnaires appear to be reliable tools for assessing specific determinants of cycling to school. Furthermore, it was found that children overestimate their abilities to perform basic bicycle skills. This suggests that objectively measuring bicycle skills is preferred to child reported skills assessment.
\end{abstract}

The importance of physical activity for health in children has been well documented $(17,25)$. However, in many countries physical activity levels in children are assumed to be too low (4) and further declining (11). Consequently many researchers have tried to identify target behaviors for increasing physical activity in children. Recently active commuting to school has been identified as an important and often underestimated target for increasing physical activity levels in children as it has the potential to integrate physical activity into daily routines (18).

Several studies investigated the determinants of active commuting to school making use of questionnaires $(7,13,17,18,25)$. Most of these questionnaires are based on concepts of socioecological models to gain insight in the role of personal, social and physical environmental determinants of active commuting to school. Despite the fact that walking and cycling to school are two different behaviors, with a different impact on health (21) and each with their own characteristics and determinants, most studies did not make a distinction between both. In addition, Hume (13) recommended that predictors of walking and cycling to school should be investigated separately in countries in which cycling to school is more prevalent. 
Understanding the different determinants of active commuting to school may be particularly important during the transition from childhood into adolescence. This critical period (10-12 years) is characterized by drop out from sports, increase of computer use and declining levels of physical activity (10,31). Furthermore, during this transition independent mobility changes (3), as children might get allowance to walk or bike to school, and children start to get more decision making power about how they travel to school.

Most of the focus within interventions on active commuting to school has been on walking, while cycling to school may have a greater potential to substitute for motorized transport because of its ability to cover greater distances at faster speeds. Furthermore cycling includes additional benefits compared with walking. First, cycling as a mode of transportation appears to be more energy intensive per unit of time than walking (21) which indicates that cycling better meets recommended intensity levels to benefit health. Secondly, cycling to school has been shown to be related to higher levels of physical fitness, while this was not the case for walking to school $(9,30)$. Thirdly, children commonly prefer cycling rather than walking (21). Finally, in some European countries cycling to school is much more common than walking to school (22). To develop effective interventions, determinants of cycling to school need to be better understood.

In the literature no studies could be located looking into specific determinants for cycling to school among children. Up to now it is unknown if basic bicycle skills of a child predict bicycling to school among 4th to 6th graders. Since road safety has been identified as an important determinant of active commuting to school $(17,25)$ and travel choices of children are strongly influenced by views of parents $(16,17)$, it is interestingly to know if bicycle skills of a child play a role in the decision of parents to allow their child to bicycle to school. Furthermore, it is unclear if the equipment of a child's bicycle is a potential determinant of cycling to school. To conclude, little is known about the role of specific cycling factors in cycling to school. Therefore a child and parental questionnaire was developed to gain insight into specific determinants of cycling to school among children. Three studies were done to evaluate the questionnaires. The purpose of the first study is to present test-retest reliability and validity of the newly developed child and parental questionnaire. The purpose of the second study is to present validity of the question on bicycle equipment in the child questionnaire. Finally, the aim of the third study was to investigate the validity of the question on basic bicycle skills in the child questionnaire.

\section{Material and Methods}

Three studies are presented: Study I represents the test-retest reliability and validity of the newly developed determinants questionnaires. Study II assessed the validity of the child reported bicycle equipment. Study III assessed the validity of the child reported scores on basic bicycle skills. All three studies are executed in Flanders, Belgium. Ethical approval was granted, by the Ethics Committee of the Ghent University. Informed consent from all participating children and their parents was obtained. 


\section{Study I}

\section{Participants and Procedure}

All 4th to 6th graders $(n=69)$ from one primary school in Flanders were invited to participate in the study, that was conducted in the spring 2010 (June). A total of 68 children (mean age $=10.51$ years; $S D=1.072 ; 39.7 \%$ girls), completed the questionnaire twice within a one week interval at school with the presence of the main researcher. Only one child was not able to complete the questionnaire at retest due to illness absence, which resulted in a response rate of $98.6 \%$. At both time points the participating children received a similar questionnaire for their parents to fill out at home. The children were asked to hand the questionnaire to the same parent at both time points and to bring the completed questionnaire back to school. The first time 66 parents filled out the questionnaire, while only 33 parents $(50 \%)$ completed the questionnaire at retest. Drop-out analyses were executed to look at baseline differences between parents that stayed in the study compared with those that dropped out. No significant differences were noted between dropouts and those who filled out the questionnaire at both time points.

\section{Child and Parental Questionnaire on the Determinants of Cycling to School}

The child and parental questionnaire were based on the literature $(7,13,17,25)$ and supplemented with questions assessing specific cycling factors. Both questionnaires were first pilot tested to identify lack of clarity of the questions and the items. Modifications were made if necessary. The child questionnaire (see Table 1) contained six sections: 1) active commuting; 2) personal factors; 3 ) attitudinal and social environmental factors; 4) barriers to cycling to school; 5) risk of cycling to school; and 6) specific cycling factors. The parental questionnaire (see Table 2) contained five sections: 1 ) active commuting; 2) personal factors; 3 ) attitudinal and social environmental factors; 4) risk of cycling to school; and 5) cognitive factors.

Active Commuting to School and Cycling During Leisure Time. Children were asked about their mode of transportation to school using the question matrix developed by Bere (6). In this matrix the children indicated how many days a week they (1) walked (2), cycled, were (3) driven by car or (4) public transport to and from school during fall, winter and spring (6). Children and parents were also asked about travel time and distance to school. In addition, parents filled out the home address. Based on the address the shortest car path distance from home to school was computed in Mappy, a free route planner on the internet. To allow comparison with other studies children were asked how many times they use their bicycle as transportation mode during a normal weekday and weekend day. The trip to/from school and cycling as a sport were excluded from this question.

Personal Factors (Bicycle Ownership, Basic Bicycle Skills and Independent Mobility). Children reported whether they owned a bicycle. They were also asked to indicate how well they had mastered thirteen basic bicycle skills on a five point Likert-type scale. These basic bicycle skills were based on existing Flemish cycling 


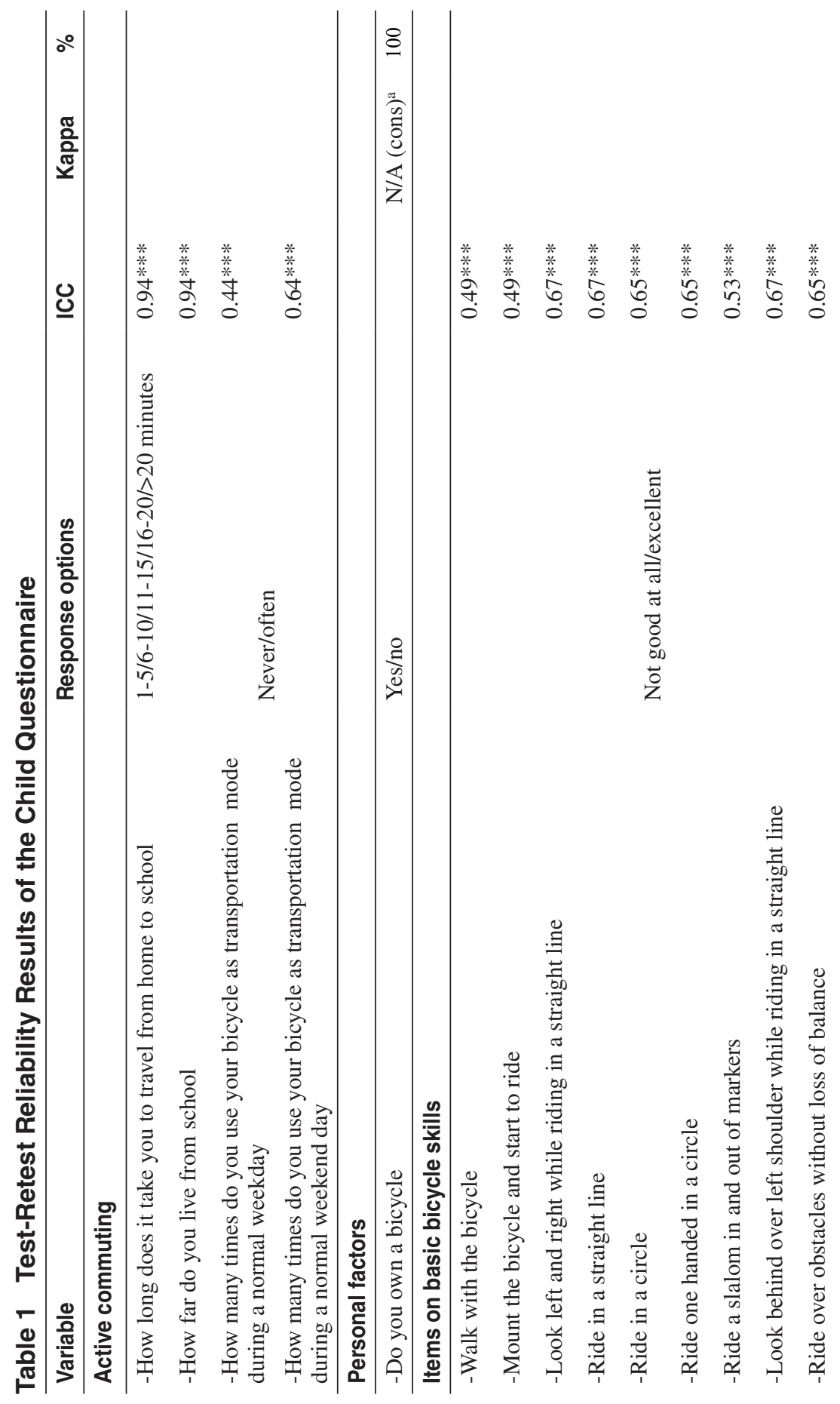




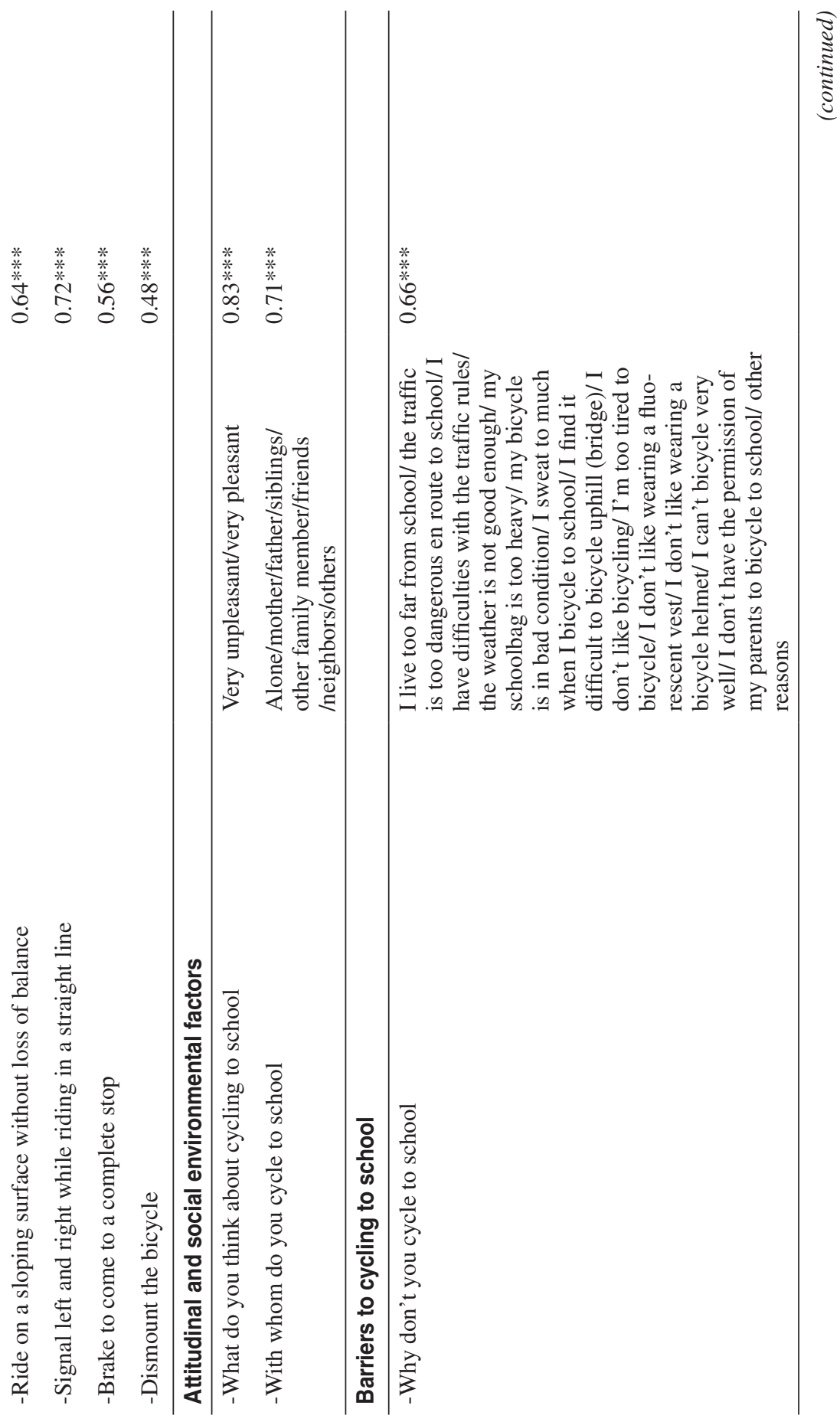




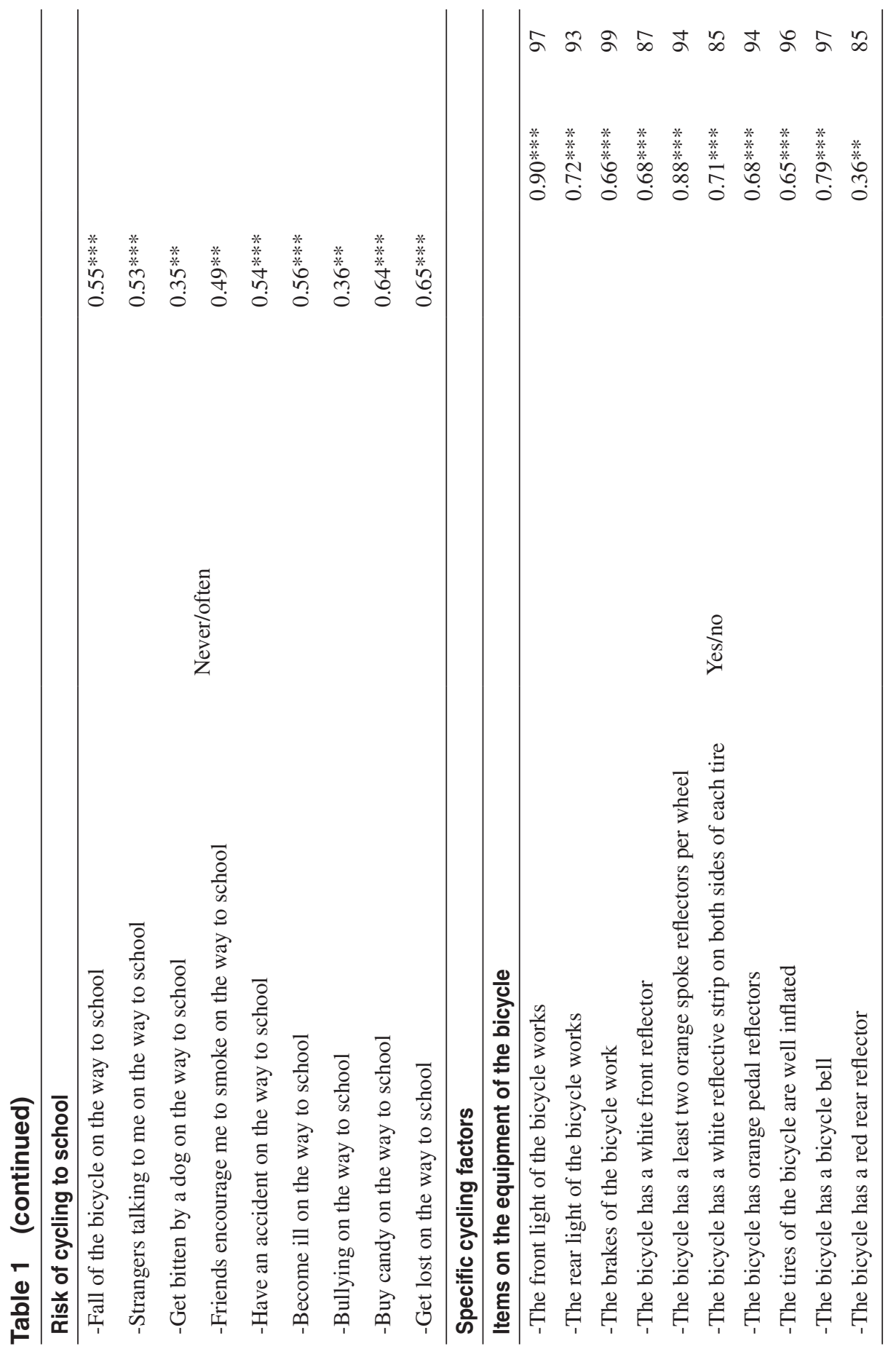




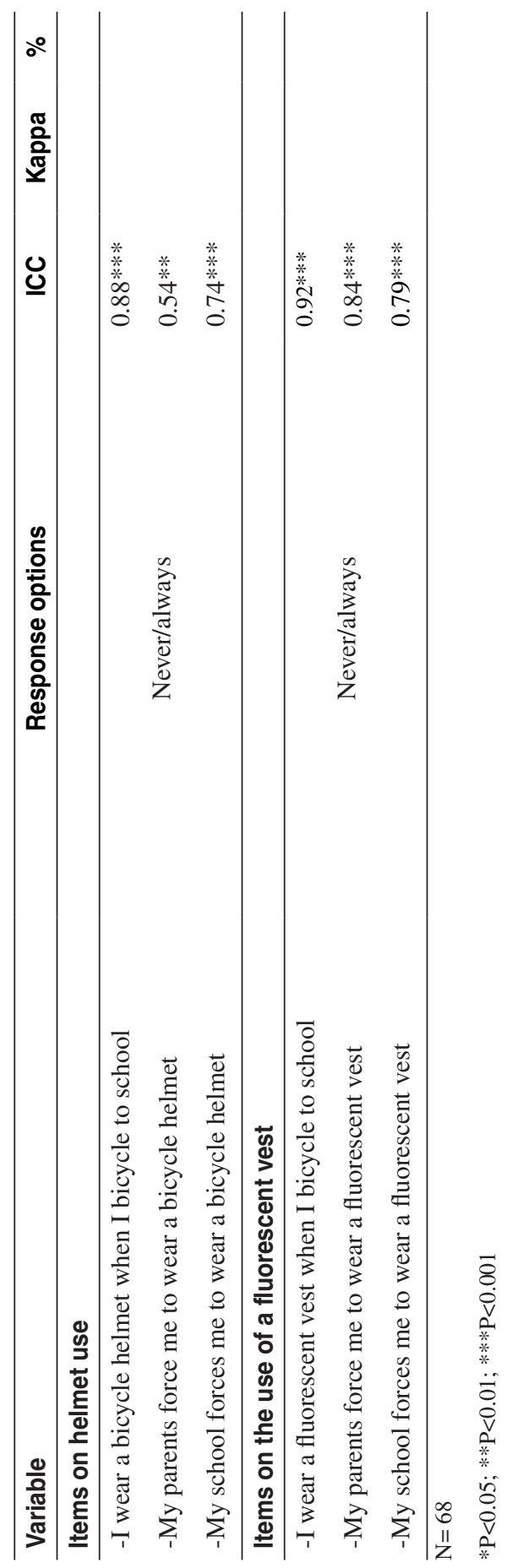




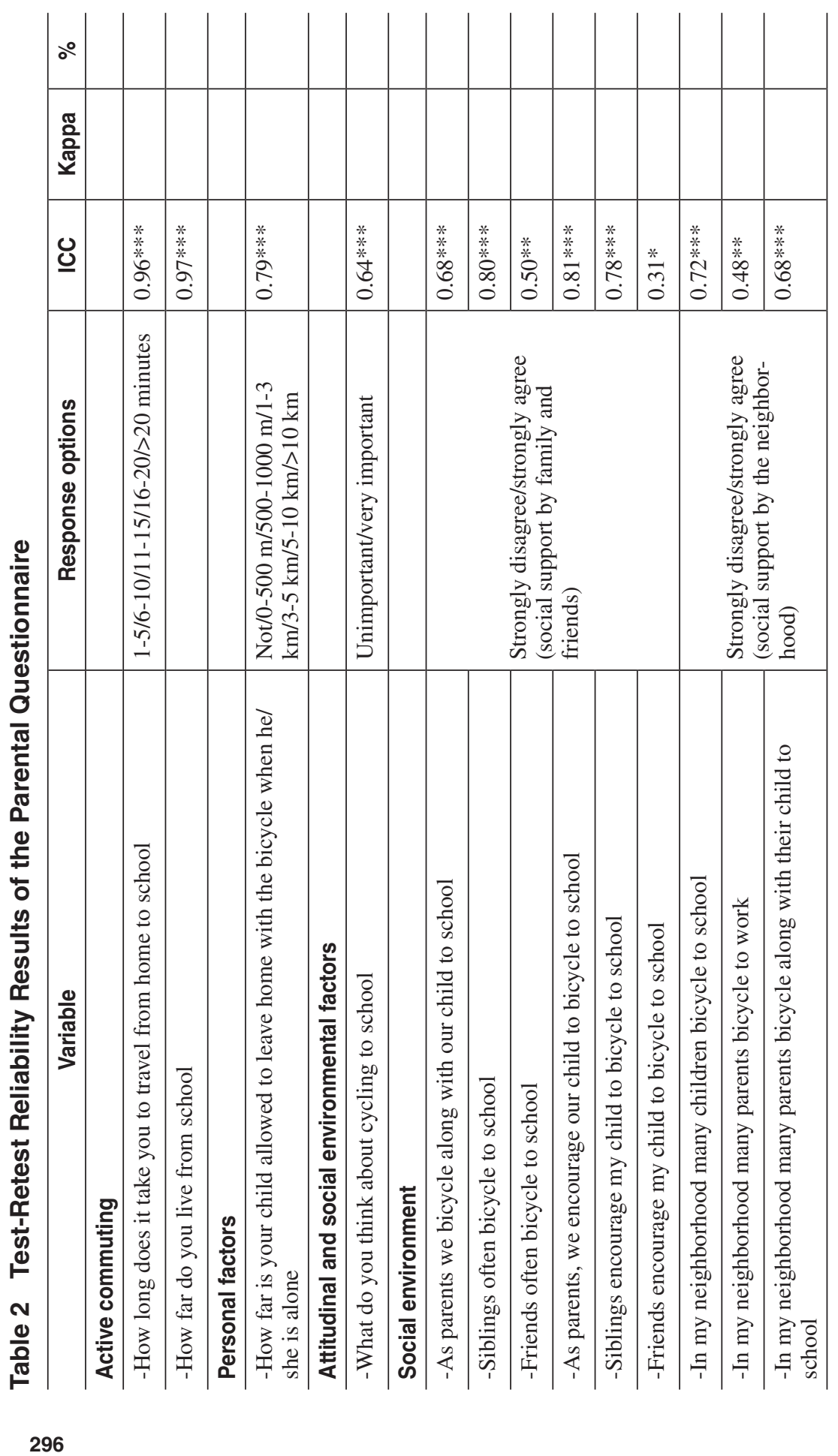




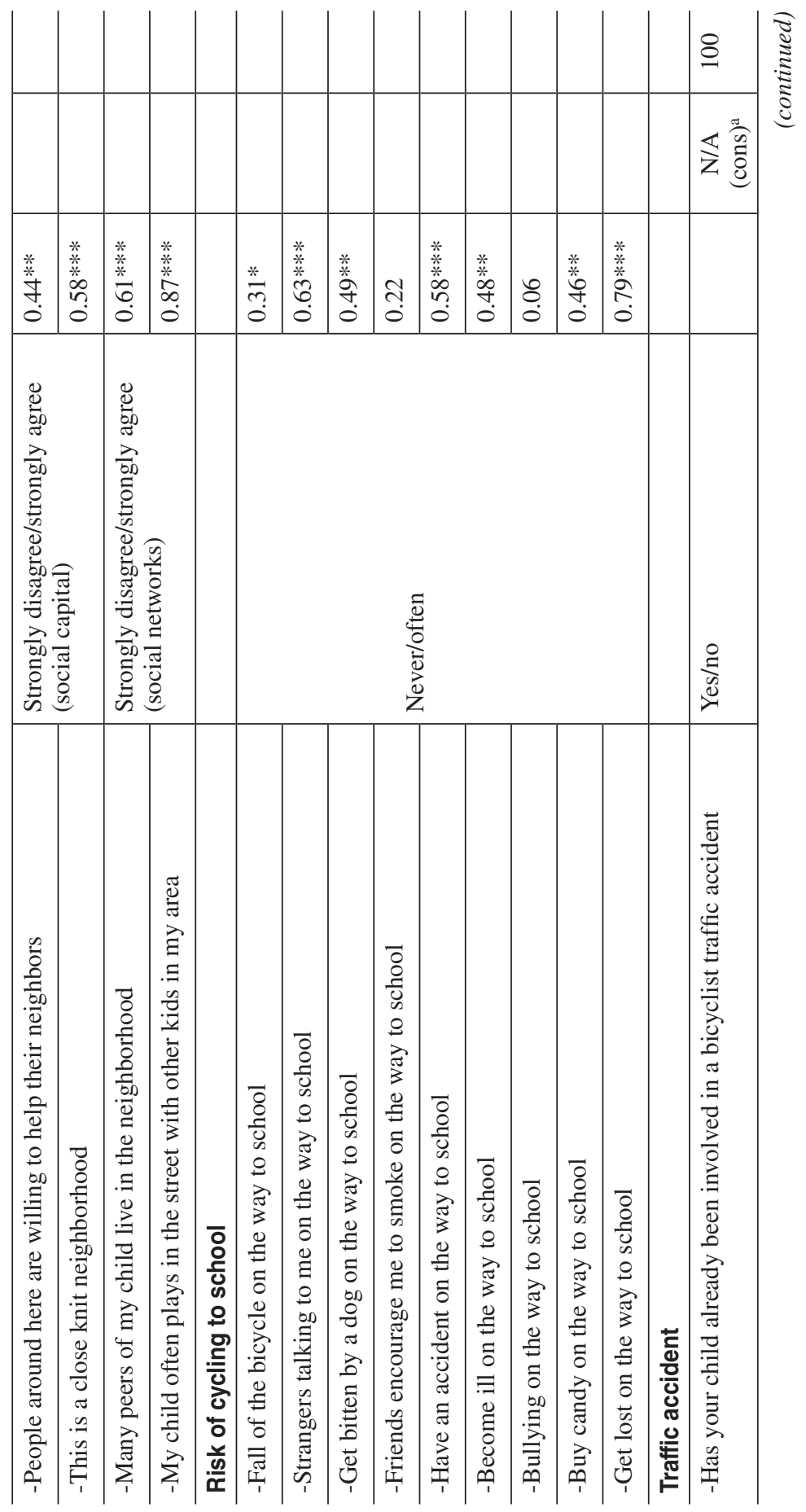




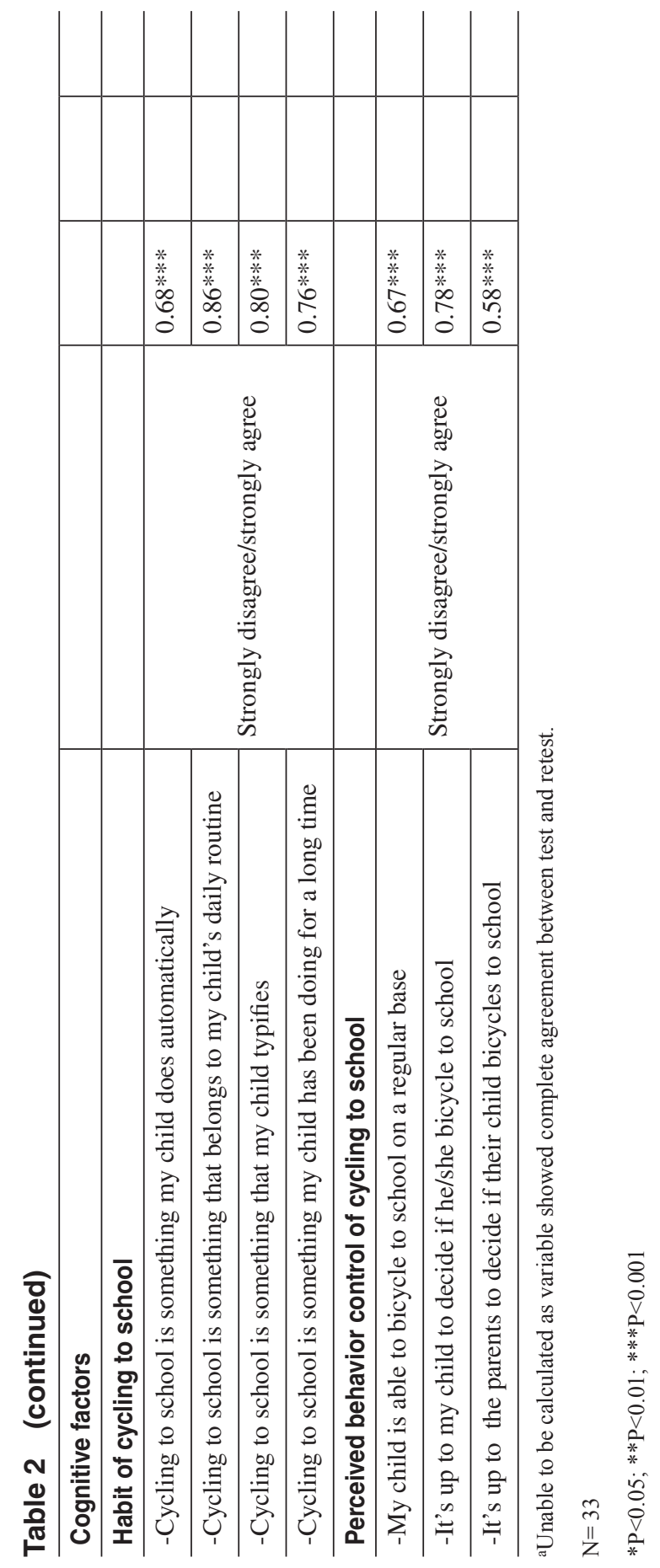


skills training programs for children $(5,23,29)$. Furthermore independent mobility of child cyclists was assessed through the parent questionnaire by the question 'How far is your child allowed to leave home with the bicycle when he/she is alone?'.

Attitudinal and Social Environmental Factors. In all participating children and parents, attitudes toward cycling to school was assessed with the question "What do you think about cycling to school?". Furthermore, children who reported cycling to school on a regular basis were asked to indicate with whom they cycle to school. In addition, parents reported their agreement, on a five point Likert-type scale, with thirteen statements regarding their perceptions of the social environment. Six items examined parents' perceptions of social support by family and friends, three items examined parents' perceptions of social support by the neighborhood, two items assessed parents' perceptions of neighborhood social networks and two items examined parents' perceptions of neighborhood social capital. These items were either newly developed or adapted from existing scales $(12,13,17)$.

Cognitive Factors (Habit and Perceived Behavioral Control of Cycling to School). Parents indicated how much they agreed or disagreed with four statements about their child's habit of cycling to school. These four items were adapted from the scale of Verplanken (27) which measures habit strength. Furthermore, parents were asked about their agreement on three statements referring to the perceived behavior control of their child to accomplish cycling to school. These three statements were newly developed.

Barriers to Cycling to School. Children who reported no cycling to school on a regular basis were asked to indicate from a list of 15 items the main reasons why they did not cycle to school on a regular basis. These items were either newly developed or adapted from a study of barriers to walking and cycling to school (2).

Risk of Cycling to School. All children and parents were asked to indicate how many times a specific risk situation could happen on the way to school if they/their child would cycle to school. These risk situations were based on a questionnaire of Terence (24), which asked parents and children to assess the likelihood of risk situations that might afflict their journey to school. In addition, parents indicated if their child had already been involved in a bicyclist traffic accident.

Specific Cycling Factors (Bicycle Equipment, Helmet Use and Use of a Fluorescent Vest While Cycling to School). Based on ten statements children reported the equipment of their bicycle. These ten statements were based on the legal requirements of a bicycle in Flanders (29). Children who reported cycling to school on a regular basis were asked some questions on the use of a helmet and a fluorescent vest while cycling to school. All questions were newly developed.

\section{Study II}

\section{Participants and Procedure}

All 4th graders $(n=35)$ from a convenience sample of two primary schools in Flanders were invited to participate in this study, which was conducted in the winter 2010 (December). A total of 28 children (response rate $=80 \%$; mean age $=9.14$ years, 
$S D=0.525 ; 42.9 \%$ girls $)$, completed the child questionnaire on the determinants of cycling to school. For the current study only the question on the bicycle equipment was used. Furthermore, children were asked to bring their bicycle to school. After completing the questionnaire in the classroom, children showed their bicycle to the researcher, who checked in detail the equipment of the child's bicycle and filled in the same question on bicycle equipment as the children did. Seven children were excluded as these children had forgotten to bring their bicycle to school.

\section{Study III}

\section{Participants and Procedure}

All 4th graders $(n=129)$ from a convenience sample of five primary schools in Flanders were invited to participate in the study, which was conducted in the winter 2010 (December). A total of 115 children (response rate $=89.1 \%$; mean age $=9.22$ years, $S D=0.559 ; 47.8 \%$ girls), completed the child questionnaire on the determinants of cycling to school. For the current study only the question on basic bicycle skills was used. Participating children were asked to bring their bicycle to school to take a bicycle skills test. Testing was performed on the playground of the school, after completing the questionnaire in the classroom. The bicycle test consisted of 13 test stations (see Table 3), examining the basic bicycle skills that were questioned in the questionnaire. There was no practice to the different bicycle tests, as children were only instructed how to perform the tests. After a training regarding the scoring procedure, three researchers scored the children on the different tests. For each test a 5-point scale was used. Speed of execution, (not) maintaining balance, (not) correctly performing the test and the fluency of execution were taken into account when scoring. Interrater reliability was found to be very good (ICC range $=0.75-0.99$ ).

\section{Statistical Analyses}

Test-retest reliability of the questionnaires (study I) was assessed by using the kappa test for agreement and intraclass correlation coefficient (ICC) along with 95\% confidence interval (CI). Ratings suggested by Landis and Koch: $0.00-0.20$ (poor), $0.21-0.40$ (fair), 0.41-0.60 (moderate), 0.61-0.80 (substantial) and 0.81-1.0 (almost perfect) were used to interpret the results. Internal consistency of scales was determined using Cronbach's alpha. Validity of child reported distance was assessed by calculating Pearson correlation ( $95 \%$ confidence intervals for $\mathrm{R}$ ) between child reported distance and shortest car path distance measured by Mappy. A detour factor, to determine the relationship between reported distance and calculated distance, was also computed. Furthermore, a kappa statistic was calculated to assess agreement for each item from the question on bicycle equipment compared with the bicycle check by the researcher (study II). Percentage of agreement for each item was also calculated (study II). Also Pearson correlations (95\% confidence intervals for $\mathrm{R}, \mathrm{CI}$ ) between the child reported scores and the scores on the practical tests, for thirteen basic bicycle skills, were calculated to assess validity of the question on basic bicycle skills (study III). Furthermore, paired sample $t$ tests were performed to examine differences between the child's skill assessment and the bicycle test scores (study III). All data were analyzed using PASW Statistics 18 and tests were considered significant at $p<.05$. 


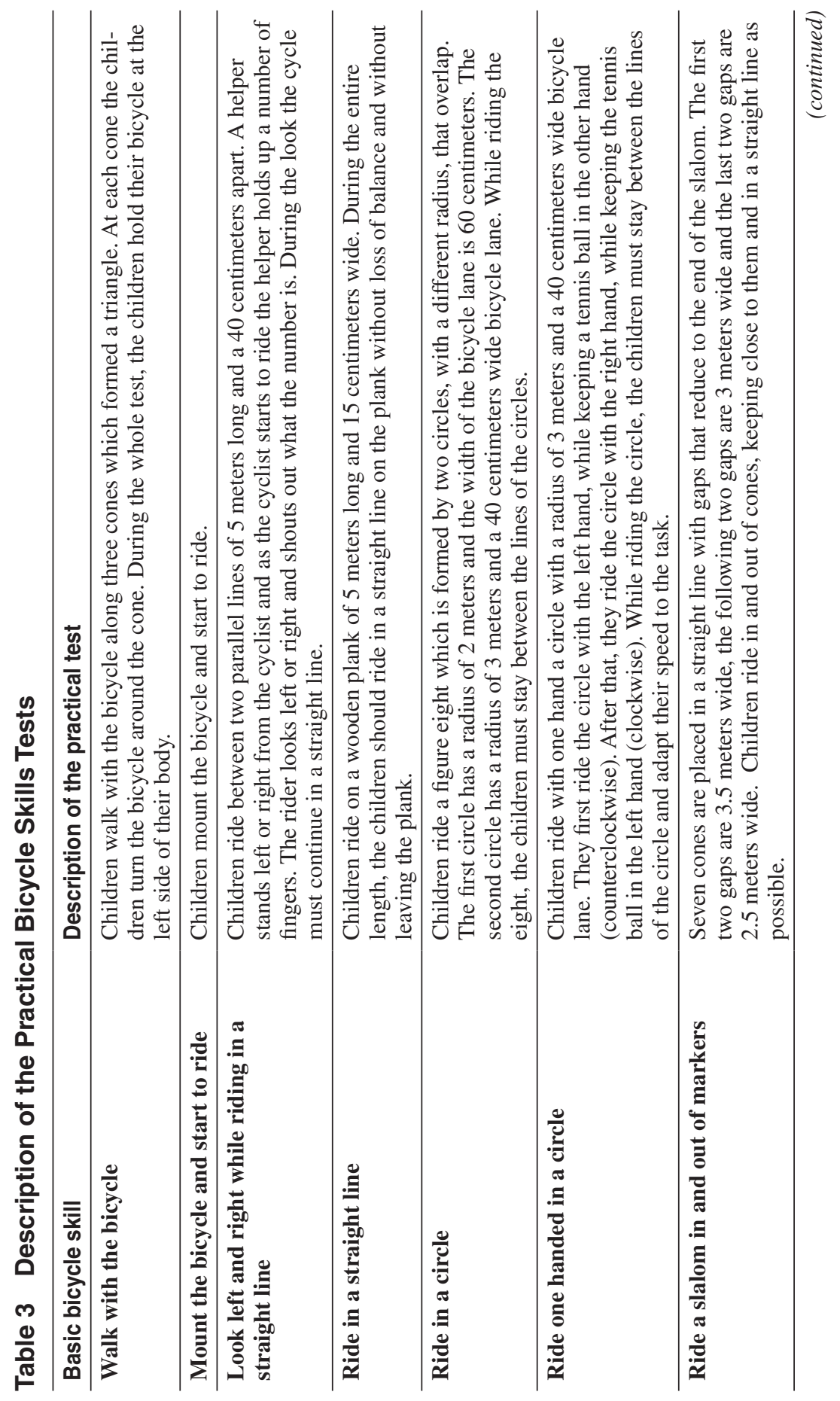




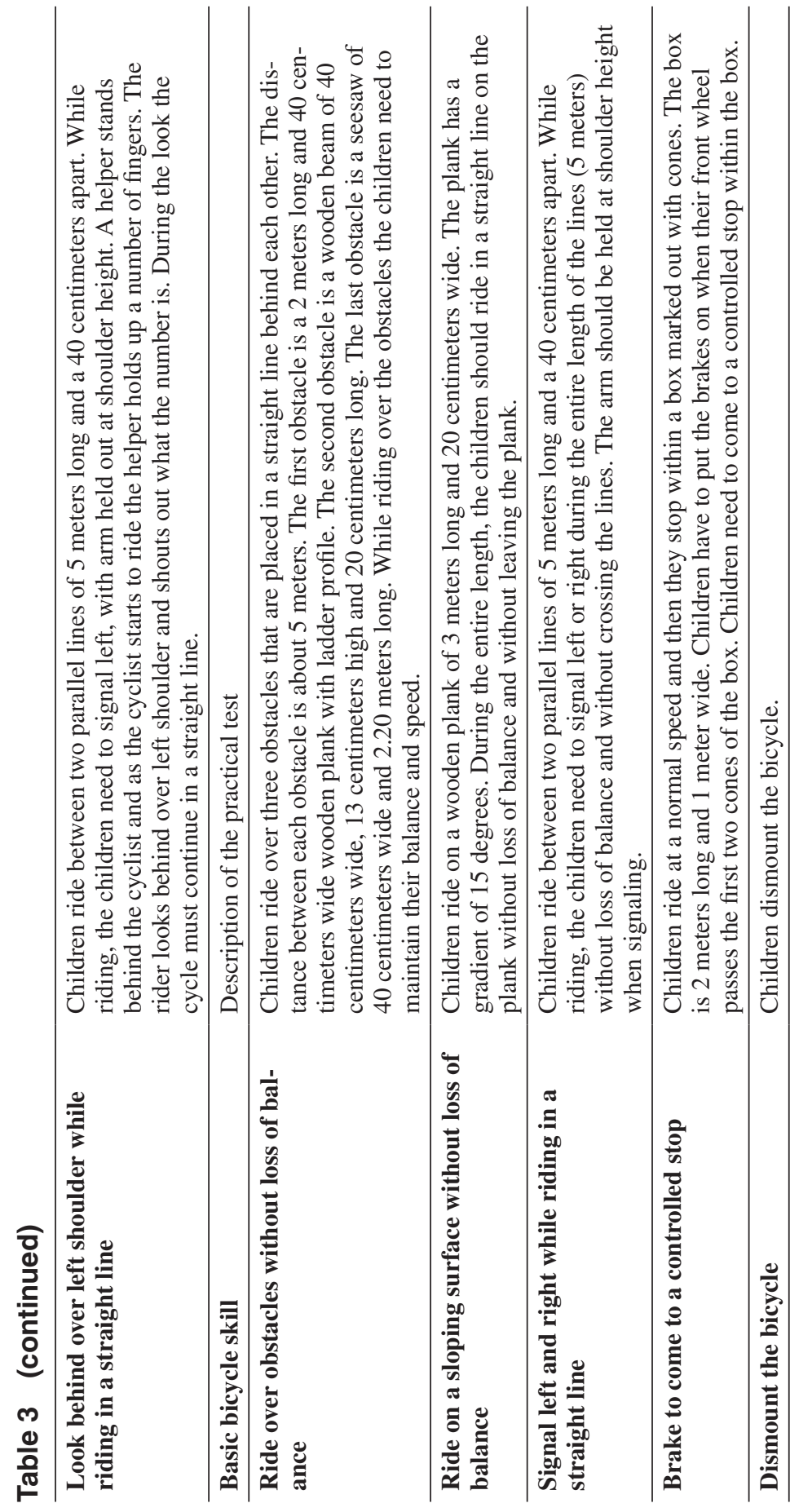




\section{Results}

\section{Study I: Test-Retest Reliability and Validity}

Test-retest reliability results of the child and parental questionnaire are presented in Table 1 and Table 2, respectively.

\section{Child Questionnaire}

Active Commuting to School and Cycling During Leisure Time. The questions regarding travel time $(\mathrm{ICC}=0.94)$ and distance $(\mathrm{ICC}=0.94)$ generated both almost perfect agreement. Furthermore, the reliability of items on cycling during leisure time ranged from moderate $(\mathrm{ICC}=0.44)$ to substantial agreement $(\mathrm{ICC}=0.64)$. The Pearson correlation between the child reported distance and the shortest car path distance was $r=.45$. Mean reported distance was $0.95 \mathrm{~km}$ lower than the mean shortest car path distance $(4.96 \mathrm{~km}$ versus $5.90 \mathrm{~km})$, resulting in a mean detour factor between the child reported distance and the shortest car path distance of 0.84 .

Personal Factors. The question on bicycle ownership generated perfect agreement as all children reported, on both time points, that they owned a bicycle. The reliability of items on basic bicycle skills ranged from moderate $(\mathrm{ICC}=0.48)$ to substantial agreement $(\mathrm{ICC}=0.72)$. Five items showed moderate agreement, while all other items demonstrated substantial agreement.

Attitudinal and Social Environmental Factors. The question regarding attitude toward cycling to school was almost perfectly reliable $(\mathrm{ICC}=0.83)$. Furthermore, the question on social support demonstrated substantial agreement $(\mathrm{ICC}=0.71)$.

Barriers to Cycling to School. The question on barriers to cycling to school generated substantial agreement $(\mathrm{ICC}=0.66)$.

Risk of Cycling to School. The items 'get bitten by a dog on the way to school' and 'bullying on the way to school' had the lowest reliability $(\mathrm{ICC}=0.35$ and 0.36 respectively). All other items on risk of cycling to school demonstrated moderate to substantial agreement. Cronbach's a was sufficient for risks of cycling to school (0.63).

Specific Cycling Factors. The reliability of items on the condition of the bicycle ranged from fair $(\mathrm{kappa}=0.36)$ to almost perfect agreement $(\mathrm{kappa}=0.90)$. The lowest reliability was found by the item 'my bicycle has a red rear reflector' and the highest reliability by the item 'the front light of my bicycle works'. Overall the three items pertaining to helmet use demonstrated moderate (ICC $=0.54$ ) to almost perfect agreement (ICC $=0.88)$. Furthermore, the reliability of items on the use of a fluorescent vest ranged from substantial $(\mathrm{ICC}=0.79)$ to almost perfect agreement $(\mathrm{ICC}=0.92)$ overall.

\section{Parental Questionnaire}

Active Commuting to School. The questions regarding travel time $(\mathrm{ICC}=0.96)$ and travel distance $(\mathrm{ICC}=0.97)$ generated almost perfect agreement. 
Personal Factors. The question on independent mobility of child cyclists showed substantial agreement $(\mathrm{ICC}=0.79)$.

Attitudinal and Social Environmental Factors. The question that assessed parents' attitude toward cycling to school generated substantial agreement (ICC = 0.64). Furthermore, the reliability of items on social support ranged from fair (ICC $=0.31)$ to almost perfect agreement $(\mathrm{ICC}=0.81)$. The two items on perceptions of neighborhood social networks generated substantial $(\mathrm{ICC}=0.61)$ and almost perfect agreement $(\mathrm{ICC}=0.87)$. For the perceptions of neighborhood social capital, "people around here are willing to help their neighbors' demonstrated substantial agreement $(\mathrm{ICC}=0.44)$. In addition, the item 'people in this area are generally get along' generated substantial agreement $(\mathrm{ICC}=0.58)$. Cronbach's a was poor for social network (0.48), but good for all other constructs of the social neighborhood $(>0.70)$.

Cognitive Factors. For the habit of cycling to school, the four items demonstrated substantial (ICC $=0.68)$ to almost perfect agreement $(\mathrm{ICC}=0.86)$. Furthermore, Cronbach's a was good for items assessing the habit of cycling to school (0.96). The reliability of items on perceived behavioral control ranged from moderate (ICC $=0.58$ ) to almost perfect agreement $($ ICC $=0.78)$. Cronbach's $\alpha$ was sufficient for items on perceived behavior control (0.62).

Risk of Cycling to School. The reliability of items on risk of cycling to school ranged from poor $(\mathrm{ICC}=0.06)$ to substantial agreement $(\mathrm{ICC}=0.79)$. One item showed poor agreement, four items showed moderate agreement and two items showed substantial agreement. Cronbach's $\alpha$ was good for risks of cycling to school (0.73). Furthermore, the question on accident involvement generated perfect agreement $(\mathrm{kappa}=1.00)$.

\section{Study II: Validity of the Question on Bicycle Equipment}

Kappa statistics for all the items on the equipment of the child's bicycle ranged from -0.07-1.00, while percentage agreement ranged from 57.1 to 100 (see Table 4). The item with the highest kappa, and the most valid item, evaluated if the brakes of the bicycle work. The least valid item, with a negative kappa of -0.07, evaluated if the bicycle has orange pedal reflectors. In addition, the items that examined the tires of the bicycle and the bicycle bell generated moderate agreement with both a 89.3 percentage agreement. All other items demonstrated fair agreement.

\section{Study III: Validity of the Question on Basic Bicycle Skills}

Seven items significantly correlated with the scores on the bicycle test, while all other items showed no significant correlation (see Table 5). The highest correlations were found for the items 'look behind over left shoulder while riding in a straight line' $(r=.47)$ and 'ride one handed in a circle' $(r=.41)$. Furthermore, for all the items except one (ride in a slalom in and out of markers) statistically significant differences were noted between child reported scores and bicycle test scores (see Table 6). 
Table 4 Validity of the Question on Bicycle Equipment

\begin{tabular}{lcc}
\hline Equipment of the bicycle & Kappa & $\%$ agreement \\
\hline The front light of my/the bicycle works & $0.39^{*}$ & 75 \\
The rear light of my/the bicycle works & $0.28^{*}$ & 78.6 \\
The brakes of my/the bicycle work & N/A (cons) ${ }^{\mathrm{a}}$ & 100 \\
My/the bicycle has a white front reflector & 0.24 & 57.1 \\
My/the bicycle has at least two orange spoke & 0.21 & 57.1 \\
reflectors per wheel & & \\
My/the bicycle has a white reflective strip on & $0.27^{*}$ & 60.7 \\
both sides of each tire & & 67.9 \\
My/the bicycle has orange pedal reflectors & -0.07 & 89.3 \\
The tires of my/the bicycle are well inflated & $0.52^{* *}$ & 89.3 \\
My/the bicycle has a bicycle bell & $0.51^{* *}$ & 75 \\
My/the bicycle has a red rear reflector & $0.33^{*}$ & \\
\hline
\end{tabular}

a Unable to be calculated as variable showed complete agreement between child reported equipment and the bicycle check.

$\mathrm{N}=28$

$* \mathrm{P}<0.05 ; * * \mathrm{P}<0.01 ; * * * \mathrm{P}<0.001$

Table 5 Validity of the Question on Basic Bicycle Skills

\begin{tabular}{lc}
\hline Basic bicycle skill & $\begin{array}{c}\text { Pearson correlation } \\
\text { coefficient }\end{array}$ \\
\hline Walk with the bicycle & 0.03 \\
Mount the bicycle and start to ride & $0.20^{*}$ \\
Look left and right while riding in a straight line & -0.02 \\
Ride in a straight line & $0.25^{* *}$ \\
Ride in a circle & 0.08 \\
Ride one handed in a circle & $0.41^{* * *}$ \\
Ride a slalom in and out of markers & $0.34^{* * *}$ \\
Look behind over left shoulder while riding in a straight line & $0.47^{* * *}$ \\
Ride over obstacles without loss of balance & 0.17 \\
Ride on a sloping surface without loss of balance & $0.34^{* * *}$ \\
Signal left and right while riding in a straight line & $0.36^{* * *}$ \\
Brake to come to a controlled stop & 0.10 \\
Dismount the bicycle & 0.02 \\
\hline
\end{tabular}

$\mathrm{N}=115$

$* \mathrm{P}<0.05 ; * * \mathrm{P}<0.01 ; * * * \mathrm{P}<0.001$ 


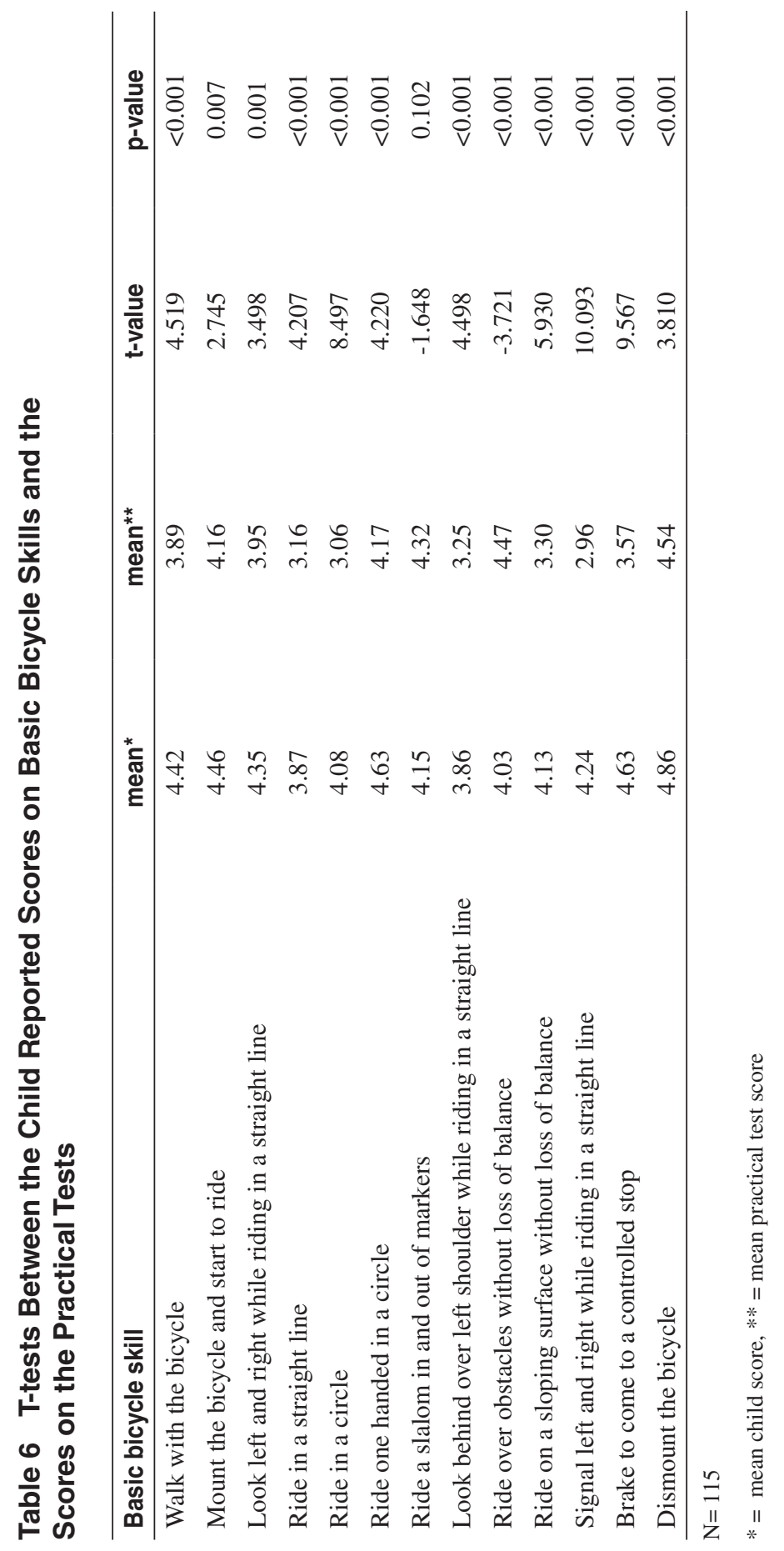




\section{Discussion}

This study evaluated the test-retest reliability of a child and parental questionnaire, developed to gain insight into the determinants of cycling to school. Validity of child reported distance, bicycle equipment and skills were also examined.

Overall the reliability of the questionnaires was acceptable with results indicating reliability ranging from moderate to perfect agreement. The highest reliability values were found for questions regarding travel time and travel distance. Since the trip to school is the same each day, it is logical that these questions are reliable. Items assessing the risk of cycling to school such as "get bitten by a dog on the way to school" and "bullying on the way to school", on the other hand, demonstrated lower test-retest reliability. This result may reflect the changeability of items on risk of cycling to school as these items may be easily overtaken by time and events.

Questions on personal factors demonstrated good reliability coefficients that ranged from moderate to perfect agreement. Furthermore, the items on basic bicycle skills generated moderate to substantial agreement. The latter finding suggest stability of items assessing basic bicycle skills.

The item on attitude toward cycling to school generated substantial and almost perfect agreement for the parental and child questionnaire respectively. Similarly, in a study of Lemieux (14) which investigated how well cognitive variables predict active commuting, the question assessing the attitude toward active commuting to school demonstrated substantial agreement $(\mathrm{ICC}=0.72)$. The items examining parental perceptions of social support by family and friends demonstrated good reliability coefficients. Furthermore, this scale demonstrated acceptable internal consistency when compared with a study of Titze (26) who found a somewhat lower Cronbach's alpha (0.68) for a similar scale investigating social support for cycling for transportation. The items on parental perceptions of the social capital showed moderate reliability. Similarly, Hume (12), who investigated the perceptions of 9-12 -year-old children on their neighborhood social capital, found moderate reliability for the item 'this is a close knit neighborhood' but higher (substantial) reliability coefficient for the item 'people around here are willing to help their neighbors'. Since these items demonstrated good reliability in both studies, they can be used in child and parental questionnaires. Furthermore, the two items on parental perceptions of the social network generated substantial to almost perfect reliability. In a study of Hume (12) similar items were used to investigate children's perceptions of the social network. The latter study reported substantial agreement for the item 'there are lots of children around to play with', but a lower (substantial) reliability coefficient for the item 'I often play in the street with other kids in my area'.

In the current study, four items of the scale of Verplanken and Orbell were adapted to measure the habit of cycling to school. These items demonstrated good reliability coefficients. In a study of Lemieux (14) the habit of using active commuting was also measured in reference to the scale of Verplanken and Orbell. Test-retest reliability result demonstrated almost perfect reliability of the scale. In addition, in a study of Verplanken (28), which measured exercising habit, high test-retest reliability was found for the scale.

In the current study, nine items assessed the perceived risk of cycling to school of children and parents. The item "get bitten by a dog on the way to school" in the child questionnaire and "bullying on the way to school" in the parental questionnaire 
had the lowest reliability coefficients. In a study of Terence (24) on perceived risks of the journey to school these two items were scored respectively on the fourth and second place by parents and 10-12 year olds as "the event the most likely to occur" and "the cause of most worry". This indicates that these two hazards are serious ones and thus play an important role regarding the perceived risk of the journey to school. Further research should examine how these potential determinants can be investigated as reports were found to be not reliable. Furthermore the item "have an accident on the way to school" generated moderate reliability both in child and parental questionnaire. Similar results were found by Carver (8) in a study assessing parents perceived risk of their child being harmed in the neighborhood and reporting an ICC above 0.60 for the item "any of your children will be knocked down while cycling in your neighborhood". In the same study the item regarding an unwelcome approach from a stranger demonstrated low reliability $(\mathrm{ICC}=0.28)$, while in our study a comparable item ("strangers talking to me on the way to school") generated moderate reliability (ICC $=0.53$ ). In general, the items assessing the risk of cycling to school demonstrated acceptable test-retest reliability.

Furthermore, in the child questionnaire an additional section referring to specific cycling factors was included. In this section the child's bicycle equipment, the use of a bicycle helmet and the use of a fluorescent vest were questioned. For the items on bicycle equipment the item "my bicycle has a red rear reflector" demonstrated the lowest reliability coefficient. All other items generated good reliability coefficients. Also items on helmet use and use of a fluorescent vest generated good reliability coefficients. This finding may suggest stability of items assessing the use of helmets and fluorescent vests for cycling to school.

In the current study, child reported distance significantly correlated with the shortest car path distance, indicating that child reported distance is a valid measurement tool. The detour factor between the reported distance and the calculated distance was below 1.0, indicating that the mean child reported distance was lower that the mean shortest car path distance. A possible explanation for the lower mean child reported distance could be found in the fact that when children report distance to school they estimate distance based on another route than the route with the shortest car path distance, which served as our reference measure in this study. Since Flanders, where the study took place, is characterized by a dense network of streets, children have several options to get from home to school. Back roads, small roads or car free roads could be part of the route to school and thus leading to lower reported distances to school compared with the shortest car path distances.

In general, fair validity was found between the child reported bicycle equipment and the bicycle check by the researcher. This suggests that children are able to report the equipment of their bicycle in a valid manner. The least valid item was the item on the bicycle's orange pedal reflectors. The fact that orange pedal reflectors require no attention from the cyclist to fulfill their task (to reflect) could be a possible explanation for the mistakes made by the children. This explanation could be strengthened by the fact that for all the items referring to the mandatory reflectors of the bicycle, lower kappa coefficients were found compared with items referring to the equipment of the bicycle that require attention from the cyclist to fulfill their task, for example the brakes or the bicycle bell. As it seems that children do not pay much attention to the mandatory reflectors of their bicycle, it is important that parents check the reflectors of their child's bicycle and take care of maintenance. 
Concerning the validity of child reported scores on basic bicycle skills, mixed results were found. Only seven of the thirteen items significantly correlated with the scores on the bicycle tests. Interestingly, the items for which no significant association were found refer to bicycle skills which at first glance seem "easier" to perform, for example "walk with the bicycle" or "dismount the bicycle". This suggest that adults (parents, teachers,. . . ) who teach children to bicycle should also pay attention to the "easier" bicycle skills. In addition, the child reported scores significantly differed from the scores on the bicycle tests for all the skills except one (ride a slalom in and out of markers). This difference demonstrated that for all the skills, except one (ride over obstacles without loss of balance), the scores reported by the child were higher than the actual test scores. This indicates that children overestimate their ability to perform basic bicycle skills. This finding is in line with other studies who found that children tend to overestimate their physical abilities $(1,1,15,19,20)$. A possible explanation for this overestimation is that children only need a relatively small chance of success to judge that they are capable of performing the skill (20). This could also explain why no significant associations were found for the easier bicycle skills, as the chance of success that children experience to perform these bicycle skills would be higher than for the other skills. Furthermore, there was no practice to the different bicycle tests, as children were only told how to perform the tests. However, if you give children the opportunity to practice the tests, children would get used to the tests which makes it impossible to measure their initial level of performance on the tests. Based on the results of this study, it seems better to objectively measure basic bicycle skills than to deal with child reported skills assessment.

\section{Limitations and Strengths}

These three studies have some important limitations. First, test-retest reliability sample size of the parental questionnaire was relatively small. Second, generalization of the validity studies is limited by the nature of the sample used which comprised children of the 4th grade only. To our knowledge, this is one of the few studies that tested and validated a newly developed child and parental questionnaire focusing on cycling to school among children. A second strength was the inclusion of specific cycling factors, that could be potential determinants of cycling to school, in the child and parental questionnaire. Finally, the inclusion of basic bicycle skills and the validation against objective testing is very innovative.

\section{Conclusion}

The questionnaires, developed to gain insight into the determinants of cycling to school generated acceptable reliability. The reproducibility ranged from fair to perfect agreement. Therefore, the questionnaires appear to be reliable tools for assessing the determinants of cycling to school among 10-12 year olds. Furthermore, child reported distance was found to be a valid measurement tool. However, most children reported a shorter route to school compared with the shortest car route. It was also found that children are able to reproduce the equipment of their bicycle correctly but that they overestimate their abilities to perform basic bicycle 
skills. This suggests that the question on bicycle equipment is reliable and valid but that objectively measuring basic bicycle skills is preferred to child reported skills assessment.

\section{References}

1. Adolph, K.E. Psychophysical Assessment of Toddlers' Ability to Cope With Slopes. J. Exp. Psychol. Hum. Percept. Perform. 4:734-750, 1995.

2. Ahlport, K., L. Linnan, A. Vaughn, K. Evenson, and D. Ward. Barriers to and facilitators of walking and bicycling to school: formative results from the non-motorized travel study. Health Educ. Behav. 2:221-244, 2008.

3. Alexander, L. The broader impact of walking to school among adolescents: seven day accelerometry based study. BMJ. 331:1061-1062, 2005.

4. Andersen, L., M. Harro, L. Sardinha, et al. Physical activity and clustered cardiovascular risk in children: a cross-sectional study (The European Youth Heart Study). Lancet. 368:299-304, 2006.

5. Belgisch Instituut voor de Verkeersveiligheid. De knappe trapper. 2009.

6. Bere, E., and L.A. Bjørkelund. Test-retest reliability of a new self reported comprehensive questionnaire measuring frequencies of different modes of adolescents commuting to school and their parents commuting to work - the ATN questionnaire. Int. J. Behav. Nutr. Phys. Act. 6:68, 2009.

7. Bringolf-Isler, B., L. Grize, U. Mäder, N. Ruch, F.H. Sennhauser, and C. BraunFahrländer. Personal and environmental factors associated with active commuting to school in Switzerland. Prev. Med. 1:67-73, 2008.

8. Carver, A., A. Timperio, K. Hesketh, and D. Crawford. Are children and adolescents less active if parents restrict their physical activity and active transport due to perceived risk? Soc. Sci. Med. 11:1799-1805, 2010.

9. Cooper, A., N. Wedderkopp, H. Wang, L.B. Andersen, K. Froberg, and A.S. Page. Active travel to school and cardiovascular fitness in Danish children and adolescents. Med. Sci. Sports Exerc. 10:1724-1731, 2006.

10. Currie, C. Young people's health in context. Health Behaviour in School-aged Children (HBSC) study: international report from the 2001/2002 survey. 2004.

11. Duncan, E.K., J.S. Duncan, and G. Schofield. Pedometer-determined physical activity and active transport in girls. Int. J. Behav. Nutr. Phys. Act. 5:2, 2008.

12. Hume, C., M. Joma, L. Arundell, J. Saunders, D. Crawford, and J. Salmon. Are children's perceptions of neighbourhood social environments associated with their walking and physical activity? J. Sci. Med. Sport. 6:637-641, 2009.

13. Hume, C., A. Timperio, J. Salmon, A. Carver, B. Giles-Corti, and D. Crawford. Walking and cycling to school: predictors of increases among children and adolescents. Am. J. Prev. Med. 3:195-200, 2009.

14. Lemieux, M., and G. Godin. How well do cognitive and environmental variables predict active commuting? Int. J. Behav. Nutr. Phys. Act. 6:12, 2009.

15. McKenzie, B.E., H. Skouteris, R.H. Day, B. Hartman, and A. Yonas. Effective Action by Infants to Contact Objects by Reaching and Leaning. Child Dev. 2:415-429, 2008.

16. Panter, J., A.P. Jones, and E.M.F. van Sluijs. Environmental determinants of active travel in youth: A review and framework for future research. Int. J. Behav. Nutr. Phys. Act. 5:34, 2008.

17. Panter, J., A.P. Jones, E.M.F. van Sluijs, and S.J. Griffin. Attitudes, social support and environmental perceptions as predictors of active commuting behaviour in school children. J. Epidemiol. Community Health. 64:41-48, 2010. 
18. Panter, J., A.P. Jones, E.M.F. van Sluijs, and S.J. Griffin. Neighborhood, Route, and School Environments and Children's Active Commuting. Am. J. Prev. Med. 3:268-278, 2010 .

19. Plumert, J.M. Relations between children's overestimation of their physical abilities and accident proneness. Child Dev. 31:866-876, 1995.

20. Plumert, J.M., and D.C. Schwebel. Social and temperamental influences on children's overestimation of their physical abilities: Links to accidental injuries. J. Exp. Child Psychol. 67:317-337, 1997.

21. Shephard, R. Is active commuting the answer to population health? Sports Med. 9:751-758, 2008.

22. Sirard, J.R., and M.E. Slater. Walking and bicycling to school: a review. Am J Lifestyle Med. 2:372-396, 2008.

23. Stichting Vlaamse Schoolsport, Mobiel 21, and Belgisch Instituut voor de Verkeersveiligheid. Meester op de fiets. 2005.

24. Terence, L., and N. Rowe. Parents' and children's perceived risk of the journey to school. Arch Beh. 4:379-389, 1994.

25. Timperio, A., and K. Ball. J. Salmonet al. Personal, family, social, and environmental correlates of active commuting to school. Am. J. Prev. Med. 1:45-51, 2006.

26. Titze, S., W.J. Stronegger, S. Janschitz, and P. Oja. Association of built-environment, social-environment and personal factors with bicycling as a mode of transportation among Austrian city dwellers. Prev. Med. 3:252-259, 2008.

27. Verplanken, B., and S. Orbell. Reflections on past behavior: A self-report index of habit strength. J. Appl. Soc. Psychol. 6:1313-1330, 2003.

28. Verplanken, B., and O. Melkevik. Predicting habit: The case of physical exercise. Psychol. Sport Exerc. 1:15-26, 2008.

29. Vlaamse Stichting Verkeerskunde. FietsIEfieTS. Mechelen, 2009.

30. Voss, C., and G. Sandercock. Aerobic Fitness and Mode of Travel to School in English Schoolchildren. Med. Sci. Sports Exerc. 2:281-287, 2010.

31. Windle, M., and J. Grunbaum. M. Elliotet al. Healthy passages. A multilevel, multimethod longitudinal study of adolescent health. Am. J. Prev. Med. 2:164-172, 2004. 